\title{
Mobility Solutions: An Empirical research on adaptability of mo- bility solutions by different enterprises with special reference to IT \& education sector in Pune city.
}

\author{
Rahul Arjun Jadhav, Vaibhav Govindjiwala \\ Assistant Professor, MBA Department, Sinhgad School of Business Studies, Pune, India. \\ MBA Department, Sinhgad School of Business Studies, Pune, India.
}

\begin{abstract}
Given the rise in small and medium businesses in India and the increase in entrepreneurship, the need for cloud management services is increasing. The growth and increased adoption of cloud computing making heavily impact and changing the way businesses are conducted in the country. Open House is quite in synchronising with cloud computing accepting market.

This research is having contribution to society like finding out Target Drunk Drivers, Exam results through mobile "SMS Examination results". This research would help to mobile solution providers to get an insight on various perceptions of small, medium \& scale enterprises and to redesign effective strategies that will enable them to increase sales.

Major objective of this research was study of mobility solution adapted by different enterprises \& their perception with specific reference to IT and Education sector in Pune region. Primary data collected with structured questionnaire from small, medium \& large enterprises from IT and education sector regarding their perception, uses \& expectations from mobility solutions. Secondary data which was mostly related to mobility solutions from white papers \& case studies collected from company website \& information related different types of mobility solutions \& trends in mobility solutions \& information of competitors collected from books and periodicals, guides, indices and websites of different mobility solution provider companies.

As Pune is one of the top most city in India \& there are different categories of IT companies \& Educational Institutes present in city so Pune area can be consider as representative of India as well as entire world. Parametric test like Chi square test are used for data analysis \& drawing results understanding perception of customers regarding mobility solutions \& their expectations.
\end{abstract}

Key Words: Mobility Solutions, Cloud computing, Open house

\section{* Need of Study}

\section{Introduction}

Given the rise in small and medium businesses in India and the increase in entrepreneurship, the need for cloud management services is increasing. Of course, Open House is providing cloud computing services over the mobile platform and the fact that it is one of the first companies to do so works highly in its favour. The growth and increased adoption of cloud computing is going to heavily impact and change the way businesses are conducted in the country. The emergence of a platform like Open House is quite in sync with our predictions and coverage and indicates towards a fast-evolving, cloud computing accepting market also, the adoption of this new technology has been early and pervasive in India across business genres. This is the reason Oracle is planning to set up base in India so as to catch-in on the growth here.

\section{* Introduction to Topic}

"Study of mobility solution adapted by different enterprises with specific reference to IT and Education sector in Pune region"

The reason for choosing this topic is that the "OPENHOUSE" is that platform which caters the need of enterprises by making them closer to their customer understandings and thus reaching out to more and more customers, also helping them with brand recalling.

One can question that how mobility solutions can help enterprises to reach out to more and more customers? Why mobile solutions only? Why not something else to come up with? The answers to these questions lay in some of data usage figures by end customers in past few years in India. Let's have a look A report by Nokia Siemens Networks has revealed a 


\begin{tabular}{|l|l|}
\hline Period- December 2011 and June 2012 \\
\hline Increase in mobile data traffic & $\mathbf{5 4} \%$ \\
\hline Increase in data traffic generated by 3G services & $\mathbf{7 8 \%}$ \\
\hline Increase in data traffic generated by 2G services & $\mathbf{4 7} \%$ \\
\hline
\end{tabular}

\section{The Path to Mobility}

Mobility will transform business value. Business application vendors know the role mobility plays in business transformation on an industry level to help them capitalize on opportunities.

For quite some time, mobility has been seen as a separate technology stack with its own unique technical challenges and solutions. That's the reason that a great number of mobile vendors on the market today developing mobile enterprise application platforms to develop unique customer solutions.

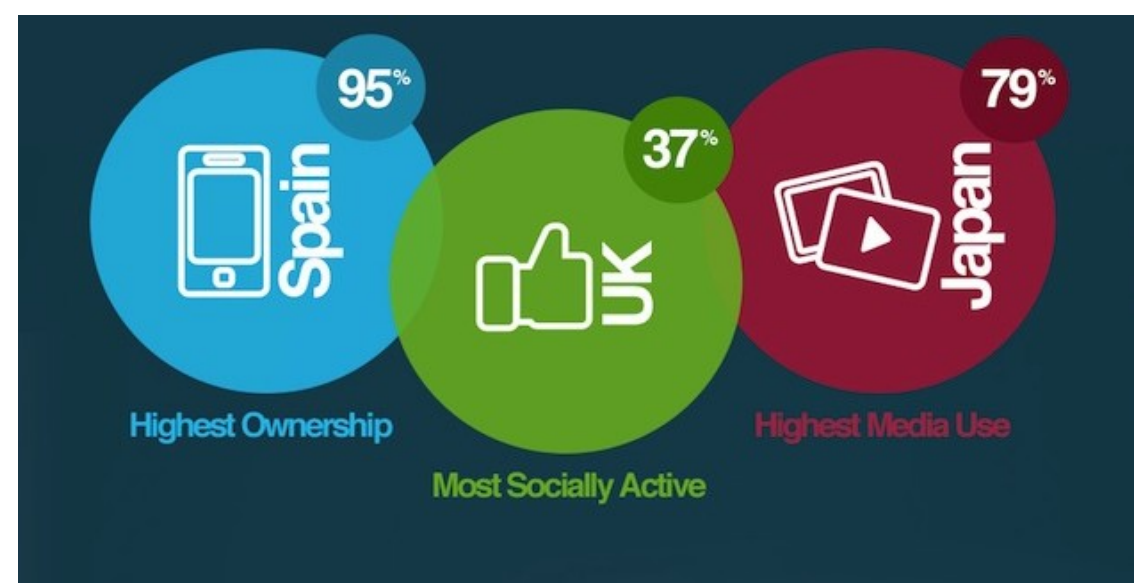

When these mobile solutions have to be integrated into the existing business applications, it is sometimes considered too difficult and too expensive because of the bolted-together platforms and an overly technologycentric approach. The business application vendor must not only understand the opportunities, but also the obstacles, surrounding what it takes to make a business mobile. So as to enable customers to realize the value of mobility by identifying outcomes and creating a clear roadmap that is led by business vision, not technology constraints.

To provide a clear path to mobility based on a platform that has mobility built in from the ground up, and a deeply rooted focus on the end-user experience. Above all, the path to greater mobility should be simple, cost-effective and incrementally rewarding. The visual breakdown of how people in Asian countries text, call, tweet and consume media on their mobiles draws on data from a recent study by Pew Research Centre and the United Nations.

Emphasising mobile phone trends in Asia, the information is broken down into mobile ownership, call usage, SMS usage, media usage and social networking. It also gives a breakdown of mobile phone ownership growth over ten years, highlighting a significant growth in developing countries. Mobile phones have transformed the way we live, work and socialise. What is even more exciting is the mobile revolution is accelerating, yet how devices are used country to country varies significantly. Usage trends help us understand how mobility is improving and simplifying healthcare and education delivery, the way we shop or bank and the options for staying in touch with family, friends and work colleagues. The path to mobility is a customer journey. Some enterprises may already have started this journey and now want to develop their business opportunities further with the help of mobility. Perhaps enterprises are about to formulate their first mobile strategy and take the first steps to making their business mobile. Wherever enterprises are in this mobile maturation process, they will always have to evolve and adapt to meet new business challenges and opportunities.

\section{State of the Mobile Industry \\ Overall Mobile User Base \\ For 2011}




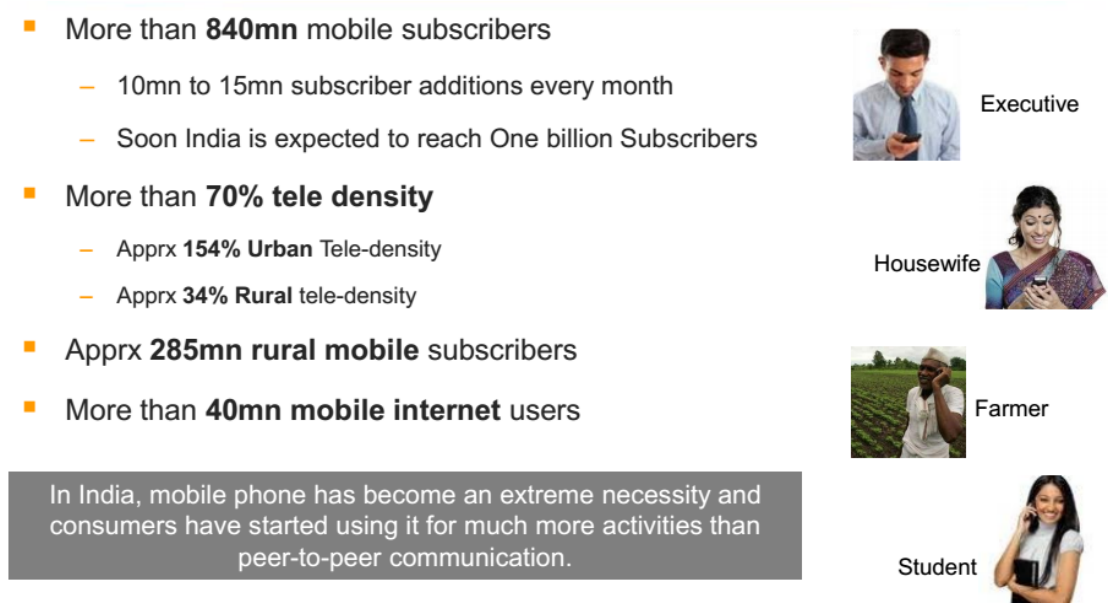

For 2012

There are 929 million mobile subscribers in India, with about 597 million urban subscribers and 332 million rural subscribers according to TRAI's press release, dated May 31, 2012.

According to the same report, the overall wireless tele density is about $76 \%$. According to a TRAI report in October 2011, only $71 \%$ of the overall subscribers were active. Further ,according to a report by GSMA, $3 \mathrm{G}$ connections are expected to grow more than 100 million by 2014, and India will be the second largest global mobile broadband market. A largeportion of this access to broadband is expected to happen through mobiles. Suffice to say that India has 'leapfrogged' from being almost a 'no-phone' nation with minuscule penetration to a 'mobile' nation with nearly a billion subscribers in a country of 1.2 billion people. According to market estimates from AC Nielsen, there are about 27 million smartphone users in urban India and still growing.

\section{Key Factors Impacting the Industry}

Over the last year, several changes have impacted the mobile industry

TRAI regulations and strict enforcement of NCPR rules caused many small Mobile Engagement Service providers to go out of business. A segment that was heavily fragmented has undergone some consolidation

Guidelines governing banking transaction message delivery requirements including use of USSD. Competition among telecom operators has driven prices of telecom bearer channels lower, removing the affordability hurdle for Small and Medium Enterprises (SMEs) / Small Office Home Offices (SOHOs). Smartphone adoption is rapidly increasing, with availability at low price points. This would allow more subscribers to access evolved Mobile Engagement solutions.

\section{Literature Review:}

In a mobility survey sponsored by IFS, which is being carried out in nine countries with a total of 455 phone interviews and web surveys with C-level executives in a number of industry verticals. Mobility as a solution has moved from being a voice-centric to a data-centric solution focusing on smartphones and tablets with access to corporate data, and this is creating a whole new set of challenges for IT managers. Mobile solutions have established themselves as an interface to email solutions, but are growing steadily as a tool for collaborative solutions, other types of communications, and for pushing information to mobile workers.

\section{The survey reveals that:}

- 59\% of the companies will increase their investments in mobile solutions. Paradoxically, $46 \%$ of the companies have no strategy in place.

- $65 \%$ of today's businesses will focus their mobile solution investments on rolling out smartphone and tablets.

- Over the nextthreeyears, 6 of 10 companies expect to invest more in mobile solutions than they did in the past.

The results indicate concrete returns on investments when implementing mobile solutions:

- Increased employee productivity and lower cost

- Remaining competitive in the industry

- Attracting and retaining talent 


\section{Driving workplace productivity through mobility}

People entering the workforce today have expectations of availability, usability, flexibility and security far beyond that of the previous generation. Mobile devices are smaller, faster and more powerful than ever before, and they're always on. These new workers expect to bring their own mobile device of choice into the workplace and add corporate email and data access as another "app" to integrate their personal and workplace communications. They expect their organizations to provide an "any data, anytime, anywhere" solution to suit their unique productivity requirements.

Accommodating these new working practices requires IT departments to create more flexible, userfocused data sharing and collaboration models vs. the centralized, standardized platform controls of the past.

The one-size-fits-all approach to mobile devices, to which organizations have grown accustomed, is now facing very real challenges due to massive demand for Google's Android, Microsoft's Windows Phone and Apple's iOS platforms. These devices allow users greater flexibility to select the applications they want to help manage their personal and professional lives. This level of diversity and choice is here to stay.

\section{There are a number of important factors to consider before making mobility an integral part of the business} process.

$>$ Tightly-controlled working locations and hours are no longer relevant for the mobile knowledge worker or field worker. The office is wherever the employee is.

$>$ Challenges will arise as best practices, policies and tools to manage corporate applications and data for desktops and laptops are extended to mobile devices.

> Mobile device management:

Mobile devices require a new set of tools for activation, asset management, troubleshooting and retirement. The tools and processes implemented for mobile device management need to be consistent and extensible across multiple mobile OS platforms. Whether managing company- or individual-owned devices, establishing a set of appropriate processes and policies is as critical as traditional systems management.

\section{$>$ Security:}

Mobile devices introduce new challenges for securing enterprise applications and data. In many cases, a user would prefer to choose and purchase their own device and wireless plan rather than use the device their employer provides. This conflict can ultimately put an organization's intellectual property and data at risk. Simply strengthening the corporate firewall is no longer an acceptable response. Successful organizations need to provide their employees mobile access to enterprise messaging, calendar, corporate directory systems, database systems and internal workgroups. Rather than saying "no," enterprises should instead manage the risk associated with the type of data and applications they require.

\section{> Mobile application architecture:}

Applications are specific to each mobile OS platform and can be sourced from app stores, via the web or pushed from enterprise IT. Enterprises need tools to build and manage applications that span multiple OS platforms, allowing varying degrees of IT and user control, and choice of which apps can be downloaded. Extending applications to customers can offer new routes to market, as well as enhance the customer experience. An environment where there is little control of the end user device presents a unique set of applications management challenges. These can be addressed by effective application development and testing.

\section{$>$ Telecom expense management:}

Expanding the mobile user community in an enterprise drives increased cost for wireless voice and data plans. To keep these costs down, enterprises should consider tools that assign users to specific voice/data plans based on their role within the organization and provide real-time controls to manage roaming.

\section{$>$ IT cost management:}

Adding more enterprise mobile devices creates new demands on mobile device procurement, activation, IT support and the help desk. The extra burden on these groups can lead to an increase in cost. To prevent this, enterprises need flexible deployment capabilities to control how new expertise, headcount, and operating cost are incurred for mobile devices.

Ideally, organizations should begin by setting out a mobile strategy and roadmap, identifying the reasons for mobility, and determining the desired outcome or benefit. Such an approach will enable identifying critical aspects and tasks in order to support the overall strategy. 


\section{How SMS Text Messaging Retains Customers \& Drives Loyalty}

Mobile advertising is still in its infancy, but that hasn't slowed the astronomical growth in businesses buying into this powerful consumer touch-point. SMS text messaging is by far one of the fastest growing sectors in mobile advertising with 9.6 trillion messages sent in 2012 alone, according to Portio Research. With mobile advertising revenue leaping to $\$ 8.9$ billion in 2012 , SMS text messaging has become an effective way to retain customers and drive long-term loyalty, especially for local businesses.

\section{Quantifying the business benefits of mobility}

To validate and quantify the business benefits of mobility solutions within large businesses, an indepth interview with large customers of Microsoft Exchange and Windows Mobile was conducted. Detailed questions about the implementation costs in deploying the software and the cost savings and other benefits realized were asked. IDC then applied our proprietary ROI methodology to the results to determine the average payback period and ROI realized by the surveyed companies.

\section{Benefits:}

IDC organized the benefits of the Windows Mobile implementation into four groups:

1. User productivity: The increased time that users have to perform business operations

2. IT operations cost reductions: IT budget capital and operations cost reductions

3. Business operations cost reductions: LOB cost reductions

4. IT staff productivity: The increased time that IT staff have for activities to support other business operations

\section{Case Studv 1:}

\section{Pharma: Field force communication}

The problem: One of India's top five pharmaceutical companies needed a cost-effective SMS application for quicker communication with its field-force and other branch offices in India. The company wanted to improve communication efficiency with/through a simple way to send messages, accept and act on responses over mobile from multiple field personnel simultaneously.

Solution: OPENHOUSE created a quick solution by configuring an interactive SMS push service. Using this service, field force could send their enquiries over SMS to a pre-defined short code and receive instant responses. Additionally, they had access to customized, real-time reports which made it easier to track and analyse their sales and marketing activities.

The outcome: The service created significantly better co-ordination among the sales teams while reducing the cost and time involved.

A sales force of about 900 individuals across India could be informed about products, stock arrivals, latest sales achievements and more, in a matter of minutes. This not only built more efficient communication, but substantially reduced the end-to-end customer acquisition costs.

\section{Case Study 2:}

\section{Automobile: Interactive promotions}

The problem: One of India's largest automobile manufacturers was looking for a simple, interactive mobile interactive platform to reach out to potential customers and generate new leads. They also wanted this platform to deliver relevant information to its customers on demand.

Solution: OPENHOUSE created a multi-keyword application for all the car models to facilitate various promotional offers. The promotions itself were carried out through SMS and radio (as a third party platform).

The company also integrated OPENHOUSE's APIs with its existing systems, to implement a 'service centre locator' application over SMS.

The outcome: The Company witnessed a threefold increase in the number of customer enquiries during the promotion period.

By providing an intuitive and interactive platform over SMS, the return on radio promotions significantly improved, even if it's a medium typically used for advertising, but with potential for lead generation.

\section{Case Study 3:}

\section{Education: Feedback application}

The problem: A well-established management learning institute, needed an interactive, mobile survey tool mobile to connect with subscribers and managers of its management training program. The aim was to SMS-enable the e-learning program on a large scale, across multiple locations within India. 
Solution: With OPENHOUSE's configurable two-way SMS engine, the institute implemented an SMS-based assessment system. A web-to-SMS solution was successfully implemented which allowed the client to configure and conduct surveys and instantly solicit responses from its subscribers.

The outcome: The institute was able to quickly and successfully augment its e-learning program with a simple, hassle free SMS solution to receive survey responses.

\section{Objectives of study:}

1. To study the benefits of mobility solutions for small, medium \& large scale enterprises.

2. To study perceptions of small, medium \& large scale enterprises on issues related to mobility solutions.

3. To study application of mobility solutions in various business functions of small, medium \& large scale enterprises.

\section{Hypothesis:}

H1: There is significant association between type of organization \& uses of application area of mobility solution.

H2: There is significant association between type of organization \& benefits received by mobility solution.

\section{* Type of Research:- Analytical Research}

\section{Research Methodology:}

Analytical Research is a research in which researcher has control over the variables. For this study researcher collected data by sending structured questionnaire through mail. This data is interpreted and suggestions are drawn.

\section{* Data type}

Primary data collected with structured questionnaire from small, medium \& large scale enterprises from IT and education sector in Pune region.

Secondary data which is mostly external data collected from books and periodicals, guides, indices and websites of different mobility solution provider companies.

\section{* Data Collection}

By sending structured questionnaire, primary data collected through mails.

\section{* Sampling}

Sample Unit: Small, Medium \& Large scale Industries from IT and Education sector operating in Pune

Population: Near about 5,000 small, medium \& Large scale Industries (IT and Education enterprises) operating in Pune.

Sample size: 180 (100 Small Scale, 40 Medium Scale, 40 Large Scale) which is stratified as 150 IT sector companies and 30 Education institutes.

Sampling Design: Probability sampling design

Sampling Technique: Stratified Random Sampling

Sample Frame: Information regarding sample units collected from Fundoo data, Chandni Chowk.com

\section{Data Interpretation and Analysis:}

\begin{tabular}{|l|c|c|}
\hline \multicolumn{3}{|c|}{ 1. TYPE OF ORGANISATION } \\
\hline & Actual & Percentage \\
\hline Small Scale & 100 & $55 \%$ \\
\hline Medium Scale & 40 & $22.5 \%$ \\
\hline Large Scale & 40 & $22.5 \%$ \\
\hline
\end{tabular}




\section{TYPE OF ORGANISATION}

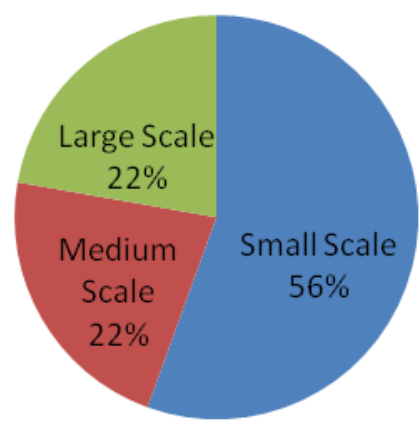

Interpretation: Out of 180 enterprises, 100 were small, 40 were medium \& 40 were large scale enterprises.

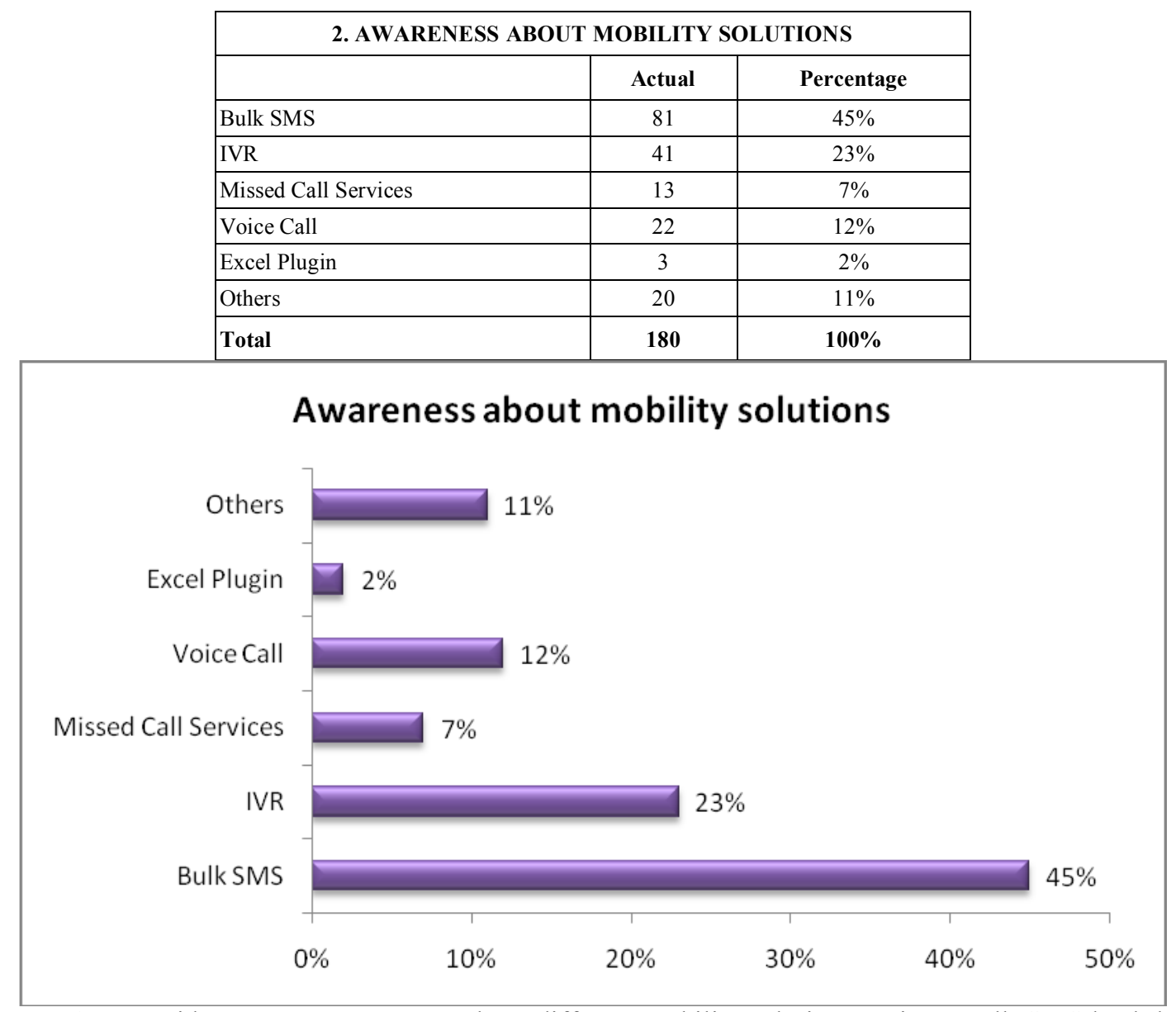

Interpretation: - With respect to awareness about different mobility solution services, Bulk SMS lead the way with $45 \%$ small, medium and large enterprises aware about, followed by Interactive Voice Response with $23 \%$. With excel plugin accounting for the lowest with $2 \%$ awareness.

\begin{tabular}{|c|c|c|}
\hline \multicolumn{3}{|c|}{ 3. MAJOR OBSTACLES IN USING MOBILITY SOLUTIONS } \\
\hline & Figure & Percentage \\
\hline Voice and data service costs & 85 & $47 \%$ \\
\hline Employee resistance to using mobile business applications & 7 & $4 \%$ \\
\hline Concerns about data, network or transaction security & 38 & $21 \%$ \\
\hline Poor performance of mobile applications & 34 & $19 \%$ \\
\hline
\end{tabular}


Interpretation: - Voice and data services cost still is the major obstacle according to small, medium and large scale enterprises accounting for $47 \%$, while $21 \%$ concerns about data, network or transaction security. $19 \%$ enterprises think poor performance of mobile applications is one of the major obstacles.

\begin{tabular}{|c|c|c|}
\hline \multicolumn{3}{|c|}{ 4. HANDLING KEY BUSINESS CHALLENGE BY ENTERPRISES } \\
\hline & Actual & $\%$ \\
\hline Quicker access to people and information to make better/faster decisions & 49 & $27 \%$ \\
\hline Increasing employee demand for mobile devices and services & 43 & $24 \%$ \\
\hline Improve customer service & 58 & $32 \%$ \\
\hline TOTAL & 180 & $100 \%$ \\
\hline
\end{tabular}

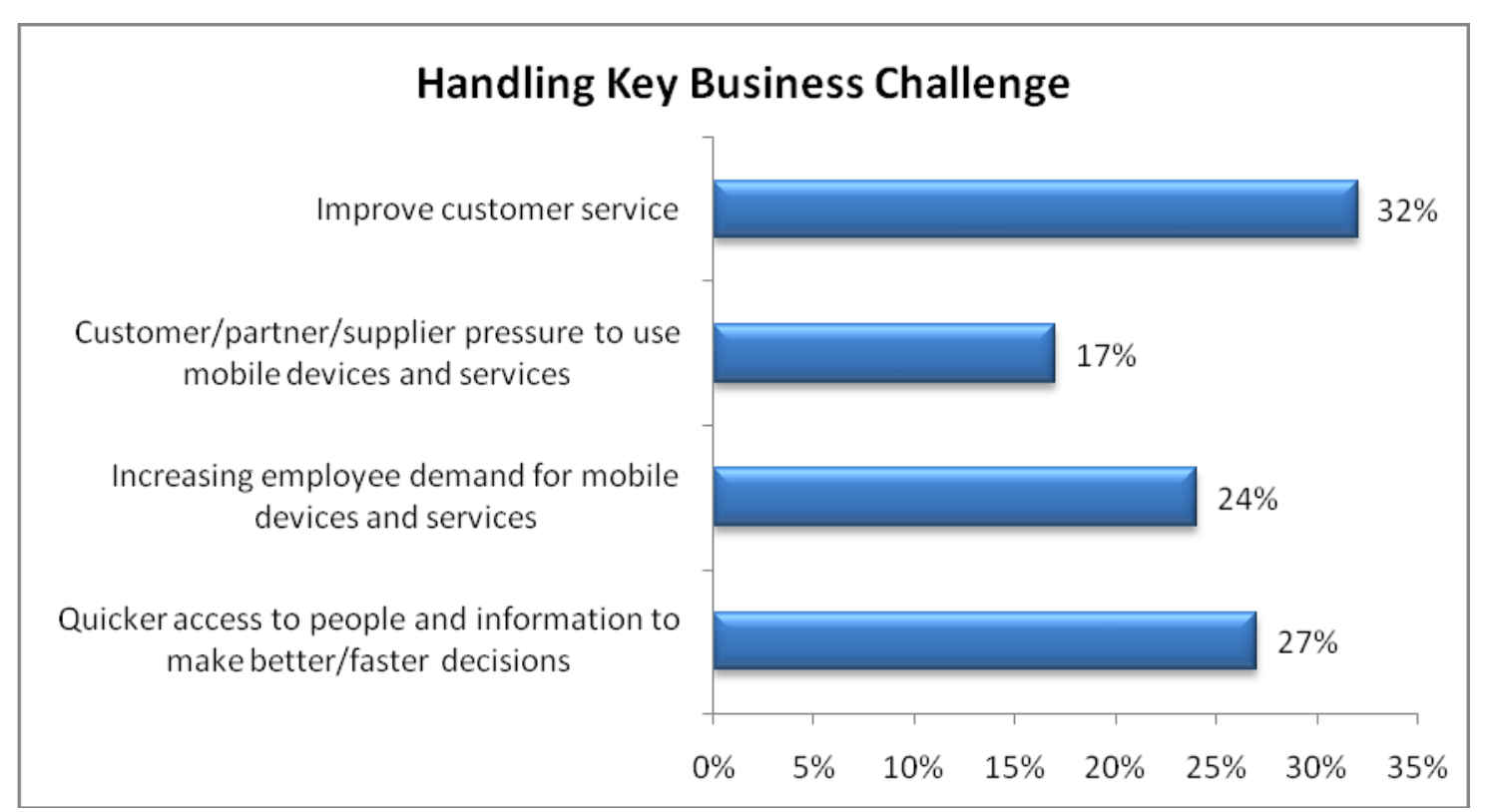

Interpretation: -From the above data it is observed that implementation of mobility solution by SMEs resulted in improvement of the customer services, as responded by $32 \%$ of the SMEs.

While $27 \%$ and $24 \%$ of the SMEs thinks that mobility solutions has helped in quicker access to people and information to make better decision and another reason being increasing employee demand for mobile devices and services.

\begin{tabular}{|c|c|c|}
\hline \multicolumn{3}{|c|}{ 5. APPLICATIONS USED AREAS } \\
\hline & Actual & Percentage \\
\hline Mobile marketing and advertising & 101 & $56 \%$ \\
\hline Customer management & 22 & $12 \%$ \\
\hline Sales force automation. & 45 & $25 \%$ \\
\hline Field service applications & 4 & $2 \%$ \\
\hline Delivery \& shipment. & 9 & $5 \%$ \\
\hline TOTAL & 180 & $100 \%$ \\
\hline
\end{tabular}

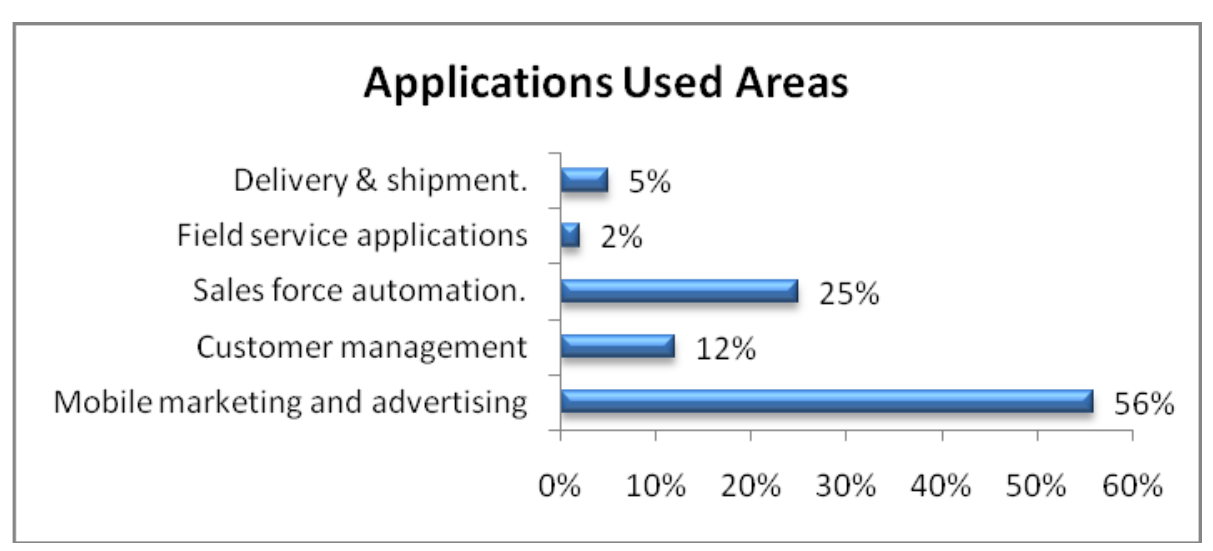


Interpretation: -More than half of the respondent SMEs uses mobility solution for the purpose of mobile marketing and advertising followed by $25 \%$ for sales force automation.

While only $5 \%$ respondent uses mobility solutions for delivery and shipment purposes, and $2 \%$ for field service applications.

\begin{tabular}{|l|c|c|}
\hline \multicolumn{2}{|c|}{ 6. BENEFITS AFTER IMPLEMENTATION } \\
\hline & Actual & Percentage \\
\hline Led to supplier and customer satisfaction. & 43 & $24 \%$ \\
\hline Increase overall productivity. & 70 & $39 \%$ \\
\hline Better utilization of resources. & 51 & $28 \%$ \\
\hline Reduced inventory levels & 16 & $9 \%$ \\
\hline TOTAL & $\mathbf{1 8 0}$ & $\mathbf{1 0 0 \%}$ \\
\hline
\end{tabular}

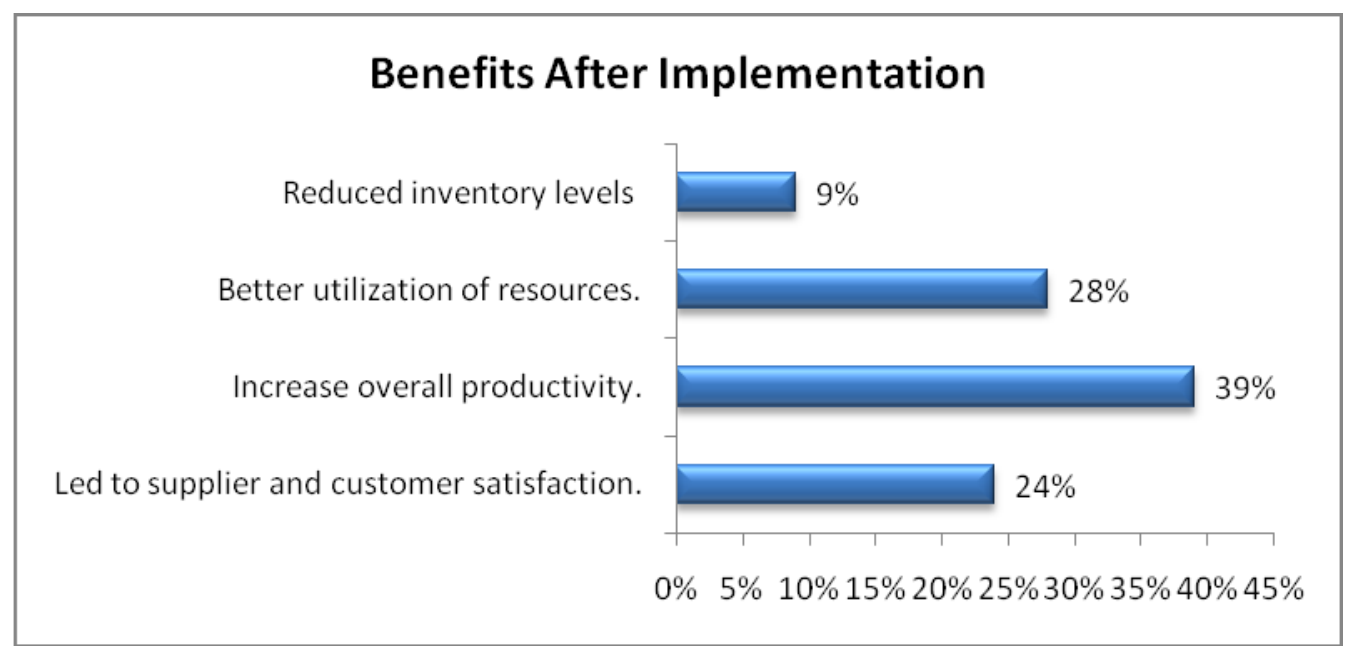

Interpretation: - 39\% of SMEs found that this solution results in increase of overall productivity. Also $28 \%$ think that it results in better utilization of resources followed by $24 \%$ SMEs which thinks that it results into supplier and customer satisfaction.

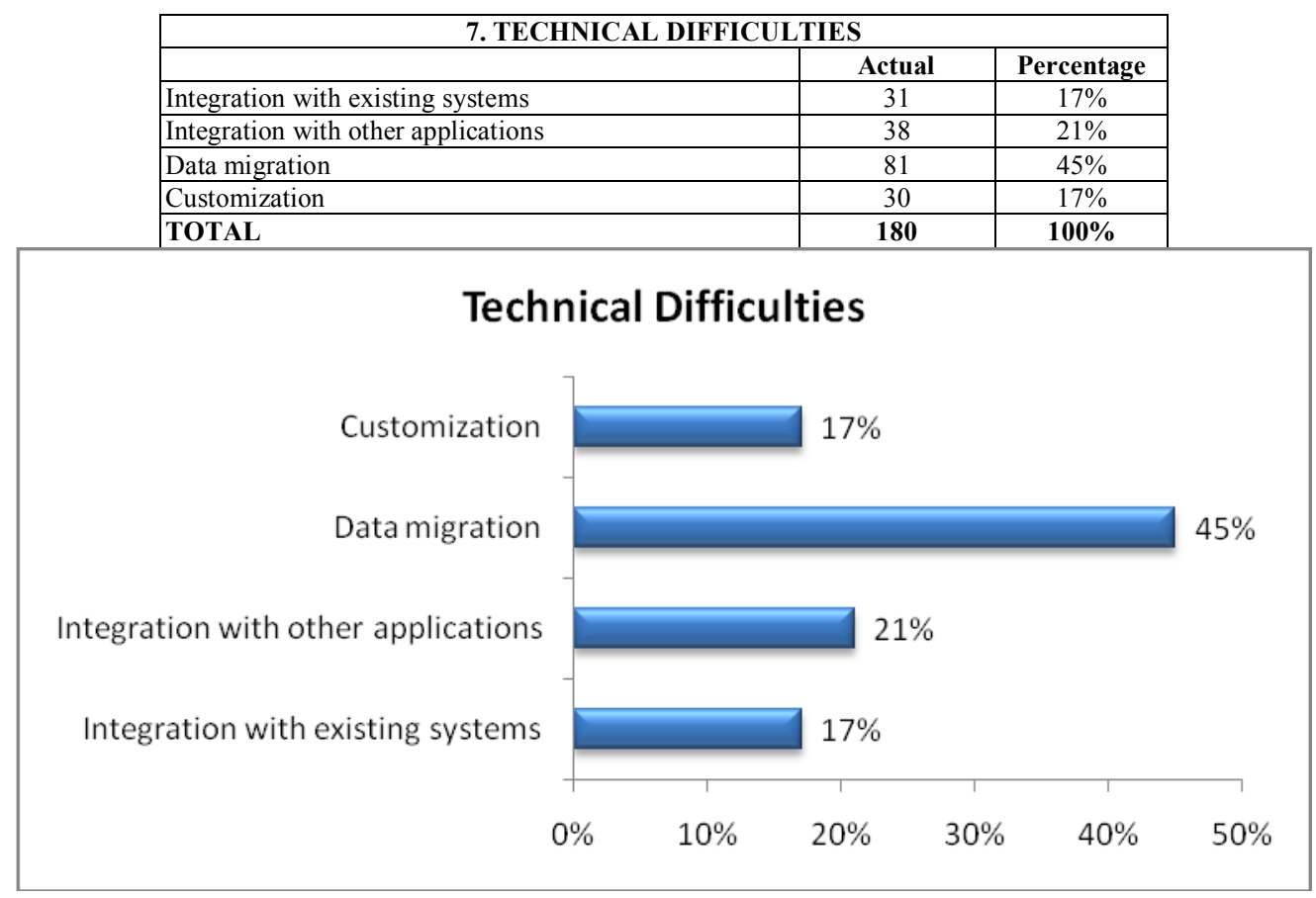

Interpretation: -Data migration is the prominent difficult in the eyes of the enterprises while implementing mobility solutions as it accounts for $45 \%$, while integration of mobile is the second most prominent technical difficult among other problems are customization and integration with existing system. 


\begin{tabular}{|c|c|c|}
\hline \multicolumn{3}{|c|}{ 8. LEVEL OF UNDERSTANDING OF USASES OF MOBILITY SOLUTIONS } \\
\hline & Actual & $\%$ \\
\hline Easy, anyone can understand but few in organizations have knowledge of it. & 63 & $35 \%$ \\
\hline Difficult, only vendor has the knowledge of it. & 50 & $28 \%$ \\
\hline TOTAL & 180 & $100 \%$ \\
\hline
\end{tabular}

\section{Level of Undersatnding}

Difficult, only vendor has the knowledge of it.

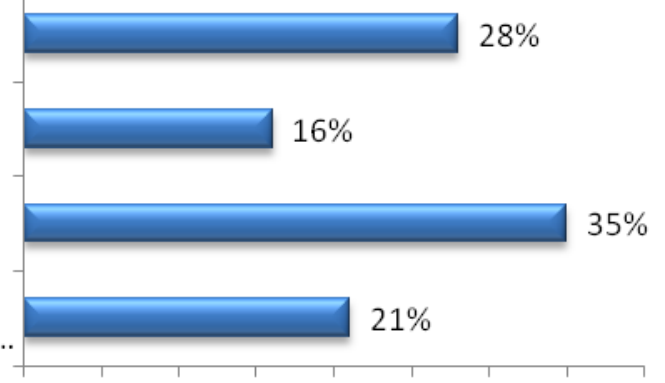

$\begin{array}{lllllllllll}0 \% & 5 \% & 10 \% & 15 \% & 20 \% & 25 \% & 30 \% & 35 \% & 40 \%\end{array}$

Interpretation: - 35\% of SMEs accept that level of understanding mobility solution and using it is easy Hypothesis Testing:

H1: There is significant association between type of organization \& uses of application area of mobility solution.

\begin{tabular}{|l|c|c|c|c|}
\hline \multicolumn{1}{|c|}{ APPLICATION AREA } & \multicolumn{4}{|c|}{ TYPE OF ENTERPRISES } \\
\hline Mobile marketing and advertising & Small & Medium & Large & Total \\
\hline Customer management & 7 & 38 & 55 & 100 \\
\hline Sales force automation. & 3 & 6 & 13 & 22 \\
\hline Field service applications & 4 & 13 & 28 & 45 \\
\hline Delivery \& shipment. & 0 & 0 & 4 & 4 \\
\hline TOTAL & 1 & 2 & 6 & 9 \\
\hline
\end{tabular}

For testing association between type of enterprises \& uses of application area of mobility solution, researcher used Chi Square test at $5 \%$ level of significance.

\begin{tabular}{|c|c|c|c|c|}
\hline $\begin{array}{c}\text { Observed Frequency } \\
(\mathrm{O} i)\end{array}$ & $\begin{array}{c}\text { Expected Frequency } \\
\text { (Ei) }\end{array}$ & $(\mathrm{O} i-\mathrm{E} i)$ & $(\mathrm{O} i-\mathrm{E} i) 2$ & $\frac{(\mathrm{O} i-\mathrm{E} i) 2}{\mathrm{E} i}$ \\
\hline 7 & 8.33 & -1.33 & 1.77 & 0.212 \\
\hline 3 & 1.83 & 1.17 & 1.37 & 0.748 \\
\hline 4 & 3.75 & 0.27 & 0.073 & 0.019 \\
\hline 0 & 0.33 & -0.33 & 0.109 & 0.33 \\
\hline 1 & 0.75 & 0.25 & 0.063 & 0.084 \\
\hline 38 & 32.77 & 5.23 & 27.35 & 0.834 \\
\hline 6 & 7.21 & -1.21 & 1.46 & 0.20 \\
\hline 13 & 12.25 & 0.75 & 0.56 & 0.045 \\
\hline 0 & 1.31 & -1.31 & 1.71 & 1.30 \\
\hline 2 & 2.95 & -0.95 & 0.90 & 0.30 \\
\hline 55 & 58.89 & -3.89 & 15.84 & 0.269 \\
\hline 13 & 12.96 & 0.04 & 0.0016 & 0.0001 \\
\hline 28 & 26.5 & 1.5 & 2.25 & 0.084 \\
\hline 4 & 2.36 & 1.64 & 2.69 & 1.139 \\
\hline 6 & 5.3 & 1.7 & 2.98 & 0.56 \\
\hline
\end{tabular}




$$
\chi^{2}=\sum_{i=1}^{k} \frac{\left(O_{i}-E_{i}\right)^{2}}{E_{i}} \quad=6.37
$$

Calculated value of Chi Square is 6.37 which is less than table value of Chi Square at $5 \%$ level of significance 15.5

Interpretation of Result: Therefore hypothesis get rejected \& it is proved that there is no association between size of enterprises \& uses of application area of mobility solution.

H2: There is significant association between type of organization \& benefits received by mobility solution.

\begin{tabular}{|l|c|c|c|c|}
\hline \multicolumn{1}{|c|}{ BENEFITS RECEIVED } & TYPE OF ENTERPRISES \\
\hline Led to supplier and customer satisfaction. & 9 & 15 & 19 & 43 \\
\hline Increase overall productivity. & 22 & 27 & 21 & 70 \\
\hline Better utilization of resources. & 12 & 11 & 28 & 51 \\
\hline Reduced inventory levels & 4 & 3 & 9 & 16 \\
\hline TOTAL & -- & -- & -- & $\mathbf{1 8 0}$ \\
\hline
\end{tabular}

For testing association between type of enterprises \& benefits received through mobility solution, researcher used Chi Square test at $5 \%$ level of significance.

Calculated value of Chi Square is 5.2 which is less than table value of Chi Square at $5 \%$ level of significance 15.5

Interpretation of Result: Therefore hypothesis get rejected \& it is proved that there is no association between type of enterprises \& benefits received through mobility solution.

\section{Findings:}

1. Bulk SMS is the mobility solution is mostly the enterprises are aware of.

2. Voice and data service cost is the major obstacle for implementing mobility solution.

3. Mobile marketing and advertising is the business application for which mobility solution is mostly used.

4. Improved customer service is the key business challenge that is best handled by mobility solution.

5. It is easy to understand use of mobility applications but very few people in organization have knowledge of it.

6. Data migration is technical difficulty while implementing mobility solutions

\section{Conclusions:}

Mobility management has emerged as a top priority within enterprises during the past 18- to 24months. Industry analysts increasingly provide more education and evaluation of solutions available to organizations seeking answers. Fortunately, companies have new choices in how they address this mission critical service they provide to their mobile workforce. However, with all these new choices comes the difficult task of deciding which the right choice for your enterprise is.

Do you want to do-it-yourself, hand it over to a third party or use Software as a Service solution? The only bad choice is to ignore the problem and suffer from overspending and waste, exposure and liability, and poor employee service. The enterprise mobility management solution enables complete and secure mobile device and data freedom. Employees gain quick, single-click access to data center. It includes beautiful productivity apps that seamlessly integrate to offer a great user experience.

It includes restriction of application access to authorized users, automatic account de-provisioning for terminated employees and selective wipe of apps and data stored on lost, stolen or out-of-compliance devices. With the Mobile Solutions Bundle, IT can meet users' desire for device choice while preventing data leakage and protecting the internal network from mobile threats.

\section{Reference Books}

\section{Bibliography:}

[1]. A Comprehensive Guide to Enterprise Mobility Jithesh Sathyan (Author), Anoop N. (Author), Navin Narayan (Author), Shibu Kizhakke Vallathai (Author)

\section{Website}

[2]. http://support.citrix.com/proddocs/topic/dws-storefront-12/dws-version-wrapper.html

[3]. http://support.citrix.com/proddocs/topic/netscaler/ns-gen-netscaler10-wrapper-con.html

[4]. http://support.citrix.com/proddocs/topic/access-gateway/ag-edocs-landing.html 
[5]. http://support.citrix.com/proddocs/topic/appcontroller-25/clg-appc-landing-page-dcon.html

[6]. http://kb1.zenprise.com/Zenprise_Device_Manager/Zenprise_Device_Manager/

[7]. http://technet.microsoft.com/en-us/library/ff622319(v=exchg.141).aspx

[8]. http://technet.microsoft.com/en-us/library/ff622319(v=exchg.141).aspx

[9]. http://www.microsoft.com/en-us/download/details.aspx?id=17206

[10]. http://i.dell.com/sites/content/business/solutions/whitepapers

[11]. http://www.huffingtonpost.com/brian-honigman

\subsection{Journals}

[12]. "Understanding Mobility Management: Trends, Priorities and Imperatives". Visage Mobile. Retrieved 24 August 2011.

[13]. "Worldwide Mobile Phone Sales Grew 35\% in Third Quarter". Gartner. Retrieved November 2010.

[14]. "Embracing Next Generation Mobile Platforms to Solve Business Problems". Computerworld Inc. IDC. Retrieved 24 August 2011.

[15]. "Sybase Warns Enterprise Over Mobile Security". Mobile Marketing. Dot Media Ltd. Retrieved 24 August 2011.

[16]. "Mobile Security a Headache for CIOs, Not a Concern for Users". eWeek. Ziff Davis Enterprise Holdings Inc. Retrieved 24 August 2011.

[17]. "Enterprise Mobility Management Goes Global: Mobility Becomes Core IT". Aberdeen Group, Inc. Retrieved 24 August 2011.

[18]. "Samsung's 90 New APIs Boost Mobile Device Management, Security". Network World. Retrieved 24 August 2011. 